\title{
Criminologie
}

\section{Le crime organisé et les pactes de non-agression : réflexions du juge Robert Cliche}

\section{Alice Parizeau}

Volume 10, numéro 1, 1977

La criminalité des affaires au Québec

URI : https://id.erudit.org/iderudit/017066ar

DOI : https://doi.org/10.7202/017066ar

Aller au sommaire du numéro

Éditeur(s)

Les Presses de l'Université de Montréal

ISSN

0316-0041 (imprimé)

1492-1367 (numérique)

Découvrir la revue

Citer ce document

Parizeau, A. (1977). Le crime organisé et les pactes de non-agression :

réflexions du juge Robert Cliche. Criminologie, 10(1), 69-78.

https://doi.org/10.7202/017066ar d'utilisation que vous pouvez consulter en ligne.

https://apropos.erudit.org/fr/usagers/politique-dutilisation/ 


\section{LE CRIME ORGANISE ET LES PACTES DE NON-AGRESSION \\ Réflexions du juge Robert Cliche recueillies par Alice Parizeau}

Dans toutes les activités commerciales, légales ou illégales, on peut relever certaines constantes et certains dénominateurs communs. Parfois, ils sont très révélateurs des mécanismes fondamentaux d'une activité donnée, parfois tel n'est pas le cas. Le juge en chef adjoint, Robert Cliche a été président de la Commission d'enquête sur l'exercice de la liberté syndicale dans l'industrie de la construction ${ }^{1}$ et en tant que tel, il a pu observer, analyser et réfléchir sur des phénomènes de ce type de la criminalité, relativement mal connu et rarement étudié sur le plan scientifique.

Une des constantes qu'on devrait étudier, remarque-t-il, c'est celle de la localisation géographique du crime organisé. Il n'établit jamais de "siège social » dans les capitales où se trouve le gouvernement. Cela est aussi vrai pour Paris, que pour Washington ou Londres, pour Ottawa ou pour Québec. Au contraire, il est particulièrement actif dans des villes telles que Montréal, Chicago ou Marseille. Pourquoi?

En fait tout se passe comme s'il y avait une entente tacite entre les dirigeants des gouvernements et la mafia pour que cette dernière opère en dehors des capitales afin de ne pas susciter les inquiétudes du public qui pourrait voir là un lien direct entre le pouvoir officiel et le pouvoir occulte. C'est une sorte de pacte de non-agression. Il n'en reste pas moins que la mafia économique est présente partout, mais ses activités, le vice organisé notamment qui se caractérise par l'exploitation d'une multitude de citoyens dont un fort pourcentage peut être considéré comme des victimes consentantes, sont systématiquement développées dans des villes portuaires, industrialisées, surpeuplées, telles que New York par exemple.

On assiste là à l'apparition d'une sorte de zones d'influences.

1. Rapport de la Commission d'enquête sur l'exercice de la liberté syndicale dans l'industrie de la construction, Québec, Editeur officiel du Québec, 1975. 
Le crime organisé est tout aussi inexistant à Québec qu'à Rome et tout aussi florissant, toutes proportions gardées, à Montréal qu'à Gênes ou à Naples. Il est facile de conclure, à partir de là, que les hommes publics et leur \& bras », la police peuvent, quand ils y tiennent, protéger certaines parties du territoire national de l'établissement du réseau du crime organisé.

Il ne s'agit pas là d'une résistance particulière de la population, mais davantage d'une forme d'action policière préventive. Ce qui importe à ce niveau, c'est bien plus la lutte contre un pouvoir de pression occulte que contre le fait criminel proprement dit.

A cet égard, l'examen de l'échelle de gravité de divers délits permet de constater que nous vivons dans une société où le crime le plus important demeure celui que commet un individu qui s'attaque à la collectivité à travers un autre individu. Les sociétés occidentales attachent une grande importance à l'homme et le code criminel détermine le produit d'un consensus. Le crime le plus grave c'est le meurtre, la vie étant le bien le plus précieux pour tous et pour chacun, ensuite le viol, résultat d'une contrainte physique où généralement l'homme qui dispose d'une force supérieure soumet à son bon vouloir la victime, soit la femme, qui par définition est physiquement plus faible. Une motivation similaire de protéger les plus faibles a été à l'origine des législations relatives au détournement des mineurs, comme à tous les phénomènes relatifs à la brutalité et à la contrainte exercée à l'égard des enfants. En quatrième place se situent les crimes de prise d'otages en vue de rançon qui également comportent la contrainte physique, puis ceux concernant l'abus de confiance, dont l'usage de faux et la fraude, puis finalement les délits de nuisance et les méfaits publics.

C'est là, dans ses grandes lignes, la gradation de la réaction d'une société organisée et civilisée à l'égard de la criminalité. Dans les sociétés dites primitives où la survivance est constamment menacée, le prix de la vie d'un individu est en quelque sorte moins élevé. Dans l'échelle de la gravité des délits, le meurtre y apparaît en cinquième ou sixième place. Chez les Esquimaux, par exemple, le plus grand crime était celui de mentir aux autres et d'inciter le peuple à aller à l'encontre de son intérêt. Le phénomène de nuisance à la collectivité était stigmatisé bien plus fortement que celui de contrainte physique à l'égard d'un individu. 
La question qu'il convient de se poser dans notre société superorganisée consiste à se demander si on ne devrait pas donner plus d'importance à une nouvelle gradation du crime. On constate, en effet, que la conception que l'opinion publique se fait de la criminalité diffère désormais de celle que reflète le code criminel. C'est ainsi, par exemple, que le vol spectaculaire d'un train postal ou des camions de la Brinks aurait été considéré par le passé comme un crime majeur, tandis que désormais, pour un fort pourcentage de citoyens, il est une sorte «d'exploit sportif ». A l'opposé, le capitaine de bateau qui ne respecte pas les règlements et qui pollue le fleuve Saint-Laurent peut faire l'objet d'une réaction punitive très forte et d'une condamnation informelle de l'opinion publique.

D'une façon globale, on peut prétendre que la crise des échelles de valeurs se traduit par l'attitude selon laquelle le crime le plus important est celui qu'on commet contre la collectivité, tandis que celui perpétré à l'encontre d'un individu ou d'une compagnie devient progressivement un simple fait divers.

C'est ainsi qu'on s'émeut de moins en moins face à la multiplicité des meurtres puisque aux Etats-Unis, les statistiques indiquent qu'on commet cent meurtres tous les jours et presque autant de viols. Exception faite des phénomènes qui impliquent un sadisme particulier et relèvent de la maladie mentale, l'opinion publique ne réagit pas.

Parallèlement, les collectivités occidentales s'impliquent bien davantage que par le passé, en ce qui concerne les cruautés commises par un État belligérant à l'égard d'un autre. Cela est vrai pour les réactions de l'opinion internationale au moment de la guerre du Viêt-Nam comme de ce qui se passe au Liban, en Afrique du Sud ou ailleurs.

Prétendre que c'est là une conséquence logique d'une meilleure information fournie autant par la presse écrite que parlée, semble superficiel. En fait, ce sont les critères du crime qui ont changé, puisque celui contre la collectivité est évalué comme beaucoup plus lourd de conséquences et inexcusable, comparativement à celui commis contre l'individu dont les motivations et les causes suscitent souvent une réaction de pitié et de permissivité à l'égard de son auteur. 
A partir du moment où les sociétés occidentales ont cessé de magnifier les valeurs telles que les faits d'armes, l'héroïsme sur les champs de batailles et les conquêtes territoriales - face à la guerre perçue non pas comme une action de la masse d'individus faisant partie de l'armée, mais comme un affrontement entre des forces dotées d'un équipement mécanique plus ou moins perfectionné - la guerre est assimilée à un crime contre la collectivité.

\section{LA CRISE DES VALEURS ET LES SANCTIONS}

L'Église catholique demeure à cet égard, la seule force qui continue à maintenir la gradation classique de la criminalité telle que rattachée au concept de la faute originelle; la collectivité, elle, prend conscience de l'évolution de l'ensemble des structures qui vont à l'encontre de cette approche traditionnelle. L'Église continue de stigmatiser à travers la notion du péché le crime individuel et de se taire souvent quand il s'agit d'un génocide. Désireuse de rester universelle et apolitique, elle ne peut que faire appel à la conscience individuelle; la société occidentale, elle, refuse de juger aussi sévèrement que par le passé le criminel, mais se prononce d'une façon beaucoup plus explicite contre les pressions des Etats plus puissants allant à l'encontre des aspirations des pays plus faibles ou moins fortunés.

Cette crise des valeurs implique une révision des politiques de sanctions que les gouvernements et les autorités législatives ne parviennent pas à entreprendre. En premier lieu, le concept même des sanctions contre les crimes commis à l'égard de la collectivité est imprécis et difficile à appliquer. En fait, pour qu'elles puissent exister, il conviendrait de créer une force supranationale capable de les mettre en pratique. Des tentatives d'organiser une telle force ont été nombreuses, mais en pratique elles se sont toutes soldées par des échecs plus ou moins retentissants. La définition des intérêts de la collectivité qu'on doit protéger est tout aussi difficile à cerner.

C'est ainsi que l'Afrique a été un continent dominé par quatre puissances à l'intérieur duquel s'affrontaient pourtant les intérêts d'une cinquantaine de pays ayant une population de 50 à 60 millions d'habitants. L'agression d'un pays à l'égard d'un autre suscitait l'intervention parallèle d'une ou de deux superpuissances qui agissaient au nom des nobles principes sans oublier pour 
autant leur propre intérêt commercial ou stratégique. Toute la notion de la justice devenait dès lors parfaitement illusoire et inapplicable.

Quand un juge condamnait à mort un voleur, il appliquait une sanction consacrée par le consensus national. Mais quand il s'agit de grands groupes qui s'affrontent, il est malaisé de prétendre que l'intervention d'une justice supranationale peut répondre à un consensus supranational. Un crime individuel avait des circonstances atténuantes qu'on pouvait évaluer à travers les causes socio-culturelles, ou les impulsions temporaires, et sanctionner au nom de l'intérêt public et de la protection des victimes éventuelles. La société pouvait légitimer son approche punitive.

A l'opposé, quand Israël veut sauver ses otages, la légitimité de cette action, absolue sur le plan théorique, est immédiatement attaquée par certains mouvements arabes qui plaident la légitimité de leurs aspirations à reconquérir un pays. Nous assistons donc, parfaitement impuissants, au phénomène de «crimes $\gg$ commis par des collectivités et des groupes, au nom des principes qu'on peut considérer comme immuables dans certains contextes et comme contestables dans d'autres.

A qui incombe la tâche de trancher et d'imposer des sanctions et quel tribunal sera en mesure de les faire exécuter?

Le crime organisé opère, qu'on le veuille ou non, en utilisant à son profit le concept de "l'intérêt supérieur », ou de la "raison de l'Etat ». A cet égard, le récent scandale dévoilé par le président de Lockheed et accusant directement le prince Bernard est assez significatif. Il s'agissait bel et bien d'une forme de criminalité impliquant des sommes importantes qui ont été soustraites par un individu à la collectivité des consommateurs, mais l'État, le gouvernement hollandais a jugé préférable de ne pas poursuivre l'enquête et de ne pas appliquer de sanctions.

Or, plusieurs intérêts étaient en jeu. La compagnie Lockheed peut être accusée, par exemple, de concurrence déloyale à l'égard de la compagnie canadienne Canadair et de bien d'autres encore, puisqu'elle obtenait ses contrats en distribuant des pots-de-vin au lieu de les «mériter», par l'excellence de sa production... En fait la seule sanction qui a été prise fut celle qui consistait à forcer plusieurs personnes haut placées à démissionner et ce ne fut pas là une décision judiciaire, mais bien davantage la conséquence 
d'une réaction de l'opinion publique. Les tractations ont eu lieu entre les représentants officiels de la grande industrie et de divers Etats, mais il est pratiquement impossible de les analyser en fonction de la participation à ces tractations des magnats du crime organisé, qui fort souvent jouent aussi le rôle d'intermédiaires et qui en échange de leurs bons services obtiennent ensuite la protection ou la non-intervention des pouvoirs publics, à l'égard de leurs propres activités; source de victimisation pour une multitude d'individus.

C'est là un autre aspect de collusion entre le péage et le pouvoir, et de son infiltration au niveau des politiques nationales et internationales. Pour qu'on puisse élaborer des sanctions, il faudrait tout d'abord qu'il y ait enquête et procès ; or le pouvoir judiciaire n'est même pas concerné puisqu'il n'y a ni plainte, ni poursuite.

\section{LE CRIME ORGANISÉ HIER ET AUJOURD'HUI}

C'est certainement là une des causes de l'impunité du crime organisé. Le scandale atteint constitue une forme de sanction pour les personnes qui détiennent une place importante dans la société; il ne touche nullement, par contre, les agents occultes qui les influencent et les compromettent. Les trois grandes armes du crime organisé, ce sont l'exploitation, l'intimidation, le chantage et elles sont intimement liées ; ce qui signifie que la victime ne peut porter plainte, ni même témoigner à postériori sans risquer, dans beaucoup de cas, d'être elle-même trouvée coupable, selon le code criminel, de complicité...

Et ce n'est guère là un phénomène nouveau. Le crime organisé existe depuis toujours et on peut remonter à l'époque de la monarchie française et du régime de l'intendant Bigaud en Nouvelle-France pour le démontrer. Ce qui est plus récent, c'est l'apparition de la puissance internationale des compagnies multinationales et le développement, souvent dans leur sillage, des réseaux parallèles et occultes. La forme de l'action du législateur à l'égard de certaines politiques des compagnies multinationales reste à déterminer, comme celle qu'on doit envisager pour rendre moins scandaleuse l'impunité des réseaux occultes.

Quand, contrairement à la loi sur les cartels, les compagnies décident de faire baisser sur le marché de Londres le prix du sucre en suscitant ainsi une grave crise économique chez certains 
pays producteurs, quel tribunal doit faire enquête et quel type de sanction sera appliqué ? Comment peut-on rendre punissables, en vertu du code criminel, des pratiques qui consistent pour certaines compagnies à brûler la récolte du café afin d'éviter ainsi la surproduction et la baisse des prix sur les marchés internationaux? Comment empêcher les cultivateurs de certains pays à faire pousser le chanvre indien afin de diminuer la consommation des drogues et des stupéfiants dans les pays occidentaux ? Des accords internationaux se sont avérés jusqu'à présent impuissants à éliminer ces formes de criminalité et on attend toujours des services douaniers et de la police une action énergique que les forces de l'ordre ne peuvent assumer sans compromettre en même temps les politiques touristiques par exemple, tout à fait vitales pour le revenu national de certains pays.

En somme, il s'agit là de diverses formes d'actes criminels qu'on ne parvient pas à stigmatiser, à recenser et à analyser de façon objective et scientifiquement valable, et à plus forte raison à réprimer à travers l'action de l'appareil de la justice.

Un autre champ d'action du crime organisé qu'on peut considérer comme relativement récent demeure celui de son infiltration à l'intérieur des structures syndicales. Dans une certaine mesure, les syndicats sont le reflet du patronat auquel ils doivent faire face. Ils s'organisent de la même façon à l'aide de moyens parfois similaires : dont le chantage, la fourberie, l'extorsion et le mépris, il va sans dire de la démocratie.

Au niveau du patronat, les décisions sont prises par quelques personnes, assises autour d'une table, qui représentent des milliers d'actionnaires.

Doit-on ou peut-on demander plus de vertus aux syndicats ?

S'ils appliquent à la lettre les règles d'une consultation pleinement démocratique, ils risquent de perdre dès le départ.

Les quelques hommes du patronat prennent des décisions au nom des milliers d'actionnaires qu'on ne consulte jamais, les délégués syndicaux ne peuvent pas consulter davantage tous les ouvriers impliqués. Les travers qu'on reproche aux syndicats nordaméricains ne sont en somme, qu'une conséquence des structures décisionnelles des multinationales. Certes, le crime organisé s'infiltre dans les tractations des multinationales et bien entendu, aussi dans celles qu'entreprennent les grandes centrales syndicales bien 
que leurs buts sont plus humanitaires et qu'ils luttent pour aider la classe ouvrière.

ه Durant la bataille de l'indexation en été 1974 », écrivent les auteurs du rapport de la Commission d'enquête présidée par le juge Robert Cliche ${ }^{2}$, "les travailleurs de la construction de Québec qui ne voulaient pas débrayer furent forcés de le faire par des fiers-à-bras. Doit-on ajouter qu'ils n'avaient jamais été consultés au préalable. Justement indignés, ils envahirent les bureaux de la Commission et - nous accordant beaucoup plus de pouvoirs que nous n'en avions - nous supplièrent d'intervenir pour que cesse cette violence. Nous avons indiqué à ces travailleurs qu'ils devaient porter plainte au ministère de la Justice. Pas un seul de ces travailleurs ne voulut le faire. Ce refus ne procédait pas de la peur. Le porte-parole du groupe résuma l'opinion de ses compagnons de travail en disant : "Le gouvernement nous a abandonnés ».

Incurie, lourdeur au niveau de l'administration, communication interministère trop cloisonnée ? Il nous a été difficile de départager les responsabilités.

Nous devons reconnaître cependant qu'il n'est pas toujours facile de poursuivre les contrevenants. Nous savons par l'expérience quotidienne de notre enquête publique que les moins d'actes criminels hésitent à raconter ce qu'ils ont vu, par peur de représailles. À ce sujet, nous partageons l'opinion émise par Monsieur le ministre Jérôme Choquette, lorsqu'il a dit devant nous :

Nous sommes aussi, parfois, devant des difficultés au point de vue preuve, parce que les témoins sont intimidés, craignent et ne veulent pas parler... Jusqu'à nouvel ordre, dans notre système, on ne condamne pas les gens sur des à peu près, des présomptions ou des conjectures.

On traverse actuellement, constate le juge Cliche, une crise de l'homme; une crise de responsabilités. Personne n'est plus responsable de rien.

Des solutions, existent-elles ?

Oui, mais elles sont bien plus politiques que judiciaires. La vigilance à l'égard des élus du peuple qui assument des pouvoirs

2. Rapport de la Commission d'enquête sur l'exercice de la liberté syndicale dans l'industrie de la construction, Editeur officiel du Québec, 1975 , p. 286 et 287. 
autant au niveau des administrations publiques que des syndicats, est un moyen infiniment plus puissant de répression du crime organisé que les législations qui figurent dans les codes criminels, qui deviennent caduques par la force des choses, puisque les victimes se taisent et qu'il est pratiquement impossible d'appliquer la loi.

L'infiltration du crime organisé dans les structures démocratiques est un fait indéniable; il n'en reste pas moins que les dictatures de gauche et de droite ne parviennent pas à l'éliminer avec plus de succès, malgré des contrôles prétendument draconiens et une répression infiniment moins respectueuse que celle des pays à démocratie libérale, des droits de l'homme. Ce ne sont donc pas, les structures socio-économiques et socio-politiques actuelles qui sont responsables de l'existence du crime organisé, pas plus que les monarchies de droit divin d'autrefois, mais les individus qui détiennent le pouvoir et se laissent impliquer dans des pactes et des ententes occultes. Ce sont aussi des citoyens qui s'émeuvent à la suite d'un fait divers, mais ne se préoccupent pas suffisamment de la chose publique.

"Seulement 364 jours se sont écoulés entre notre assermentation et la remise de ce rapport ${ }^{3}$, , avait écrit le juge Robert Cliche. "Nous n'avons pas perdu de temps, seulement des illusions. En effet, au départ, nous croyions rencontrer des hommes pleinement humains, beaux dans leur grandeur comme dans leurs faiblesses. Nous étions naïfs. Bien sûr que nous avons rencontré beaucoup d'honnêtes citoyens qui besognent durement et espèrent en des "lendemains qui chantent». Ces hommes étaient pour nous à la fois guide et inspiration. Mais au menu quotidien de nos audiences, nous avons trop souvent été placés en présence de fraudeurs, d'escrocs et de canailles. Et pourtant, nous n'avons jamais perdu espoir. Nous croyons que les travailleurs de la construction, les chefs d'entreprise et les agents publics intéressés à la construction sont capables de trouver en eux de nouvelles raisons de travailler ensemble, dans une paix retrouvée. Il s'agit maintenant pour eux de le vouloir $\gg$.

Approche optimiste qui est toujours celle du juge Robert Cliche. Approche qui indique clairement que la lutte contre les agissements du crime organisé incombe à tous les citoyens, par

3. Voir la p. 5 du rapport déjà cité. 
opposition à celle contre la criminalité des individus prévue dans les codes et réprimée par des lois qu'on peut appliquer à travers l'action de la police, de l'appareil judiciaire et des services de resocialisation... 